\title{
The Element Disturbed Equation of Dissimilar Material for The Analysis of Duality Disturbance
}

\author{
Shoulong Zhang ${ }^{1, a}$, Liugang Du ${ }^{1, b}$, Yanbin Huang ${ }^{1, c}$, and \\ Guangwu Zhang ${ }^{1}$, Jinhe Gao ${ }^{1,2}$, Weiling Huang ${ }^{1}$, Xiang Tao ${ }^{1}$ \\ ${ }^{1}$ School of Architecture and Civil Engineering, East China Institute of Technology, China \\ ${ }^{2}$ School of Civil Engineering, Tongji University, China

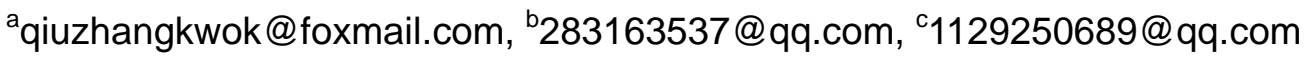

Keywords: single material element. duality disturbance equation

\begin{abstract}
For single material element, the same material element is comprised of Relatively Non-Disturbed(RND) state and Relatively Complete Disturbed (RCD)state is considered. The ratio of the two kinds of state changes with the loading level. The affection of the interaction between RND state and RCD state to the apparent behavior of $F^{0}$ is considered in the analysis of equilibrium equations. The key point of the method is that the response of RND state and RCD state can be defined by the apparent behavior of same material element. But the theory of duality disturbance can also be applied to the material element that is comprised of two(or more than two ) kind of materials. ${ }^{[3-5]}$
\end{abstract}

\section{Introduction}

Concepts duality disturbance state can not only apply to similar material elements, but also extend to the material element that is comprised of two(or more than two ) materials. This has important practical significance in engineering application. As long as we stipulate the apparent response of these materials as the reference state, these materials' responses can be described on the base of the continuum theory(e.g. The elasticity and plasticity theory).And the behaviors of every kind of material elements can perform as RND state and RCD state. As shown in Figure 1. The stress-strain curve $1 \& 2$ in figure $1(\mathrm{a})$ is the scheme of two elastic material under reference state. The apparent behavior $\sigma^{0}$ is also performance linear elasticity; as shown in figure 1(b),the stress-strain curve 1 is the linear elasticity. Material under the reference state, and the stress-strain curve 2 is the elastic-plastic material under reference state which Apparent behavior $\sigma^{0}$ performance elastic-plastic. ${ }^{[5-10]}$

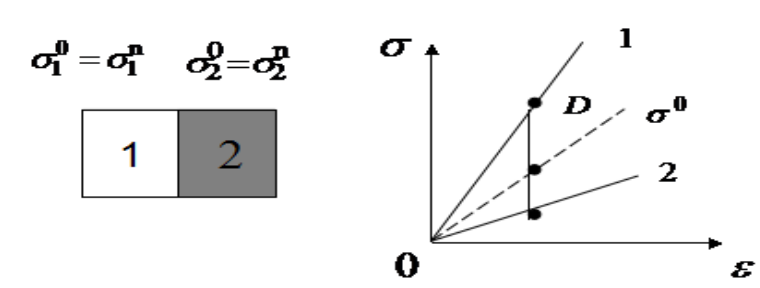

(a)
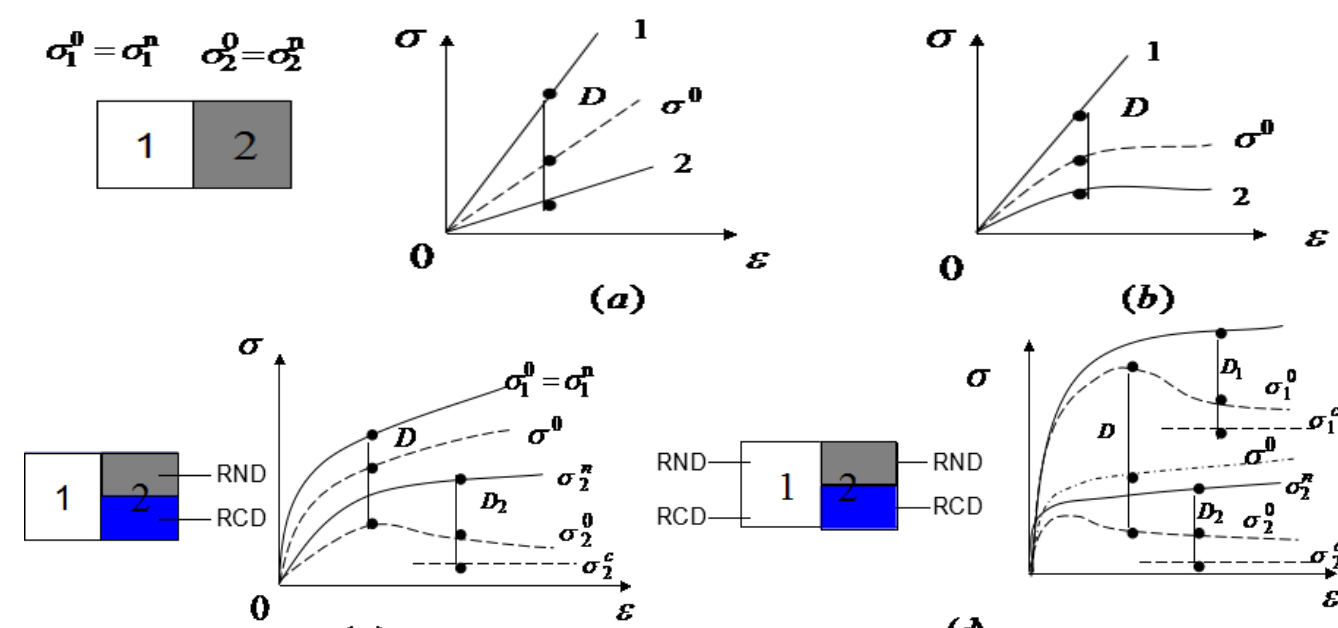

(c)

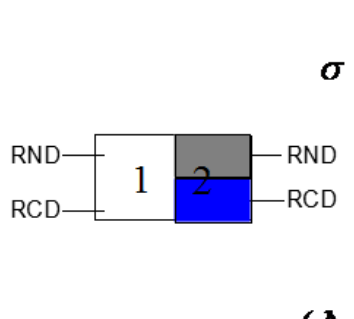

(d)

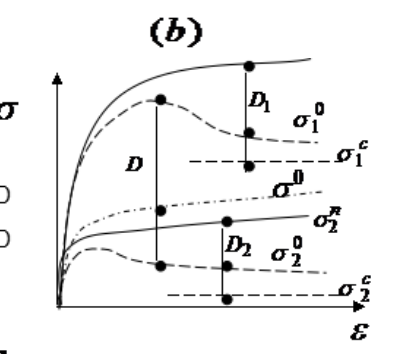

Fig.1 relatively non-disturbed state

The two material under reference state in Figure 1(a),1(b) did not related to the Fully adjustable 
state. The material 2 in figure 1(d) is involved the microcrack growth which leading the response of softening with RND state and RCD state.Figure 1 (d) shows the two materials, Each materials exhibit softening and involves RND state and RCD -state.${ }^{[3-5]}$ For the material element of different material compositions, It is necessary to evaluate each kind of material of the apparent behavior. Also on a combination of state of the apparent behavior assessed, Also to evaluate the combination state apparent behavior $\sigma^{0}$. The method of assessment is to dissimilar materials as a reference state, And then export binary perturbation equation. Such as, consider first figure 1 (a), mixed apparent stress Linear elastic material 1 and materials 2 mixed apparent stress $\sigma^{0}$ from formula(1) expression

$$
\sigma^{0}=(1-\bar{D}) \sigma_{1}^{0}+\bar{D} \sigma_{2}^{0}
$$

Formula (1) in $\bar{D}$ according to the material 1 and material 2 apparent stress is expression

$$
\bar{D}=\frac{\sigma_{1}^{0}-\sigma^{0}}{\sigma_{1}^{0}-\sigma_{2}^{0}}
$$

The quoted parameter $\bar{D}$ present a ratio means for the disturbance, but its definition is different from the disturbance in the same material unit.according to formula (1) ,Linear elastic material 1 and 2 of the mixture of apparent stress also is linear elastic.As shown in figure 1 (b) and figure 1 (a) ,the difference only lies in the material 2 for elastic-plastic materials,so the type (1) and type (2) remains valid to the figure 1 (b).Next consider Figure 1 (d),each figure reference material states that have shown micro crack and softening behavior and involves RCD state. The apparent behavior of each material can be written as

$$
\begin{aligned}
& \sigma_{1}^{0}=\left(1-D_{1}\right) \sigma_{1}^{\mathrm{n}}+D_{1} \sigma_{1}^{c} \\
\sigma_{2}^{0}= & \left(1-D_{2}\right) \sigma_{2}^{\mathrm{n}}+D_{2} \sigma_{2}^{c}
\end{aligned}
$$

Thus according to the formula ( 1 ), there are

$$
\begin{aligned}
& \sigma^{0}=(1-\bar{D})\left[\left(1-D_{1}\right) \sigma_{1}^{\mathrm{n}}+D_{1} \sigma_{1}^{c}\right]+\bar{D}\left[\left(1-D_{2}\right) \sigma_{2}^{\mathrm{n}}+D_{2} \sigma_{2}^{c}\right] \\
& \sigma_{\mathrm{ij}}^{0}=(1-\bar{D})\left[\left(1-D_{1}\right) \sigma_{1 i j}^{\mathrm{n}}+D_{1} \sigma_{1 i j}^{c}\right]+\bar{D}\left[\left(1-D_{2}\right) \sigma_{2 i j}^{\mathrm{n}}+D_{2} \sigma_{2 i j}^{c}\right]
\end{aligned}
$$

The above results can be applied to the unit.

\section{Equation of duality disturbance of porous materials}

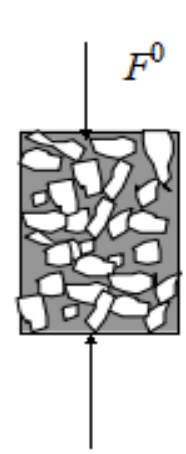

(a)

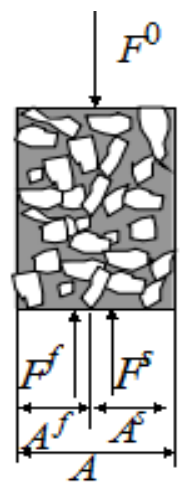

(b)

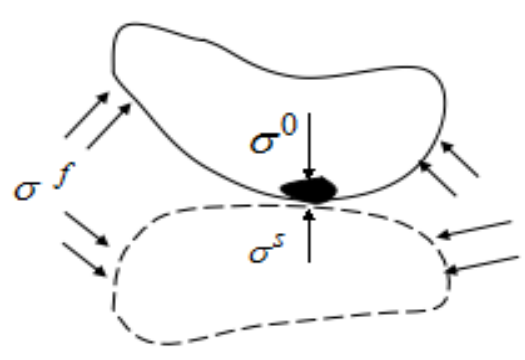

(c)

(a)Porous material and aggregate to contact

(b) the balance of contact force, fluid pressure and actual pressure

(c) The apparent stress, contact stress and fluid stress

Fig.2

Consider a fluid saturated porous materials (such as soil), As shown in figure 2 (a), there are the external force or full pressure $F^{0}$ on the material unit, which thansfer the force $F^{f}$ and $F^{s}$ through the liquid area and solid aggregate contact area, as shown in figure 2 (b), and by the force equilibrium condition 


$$
F^{0}=F^{\mathrm{f}}+F^{s}
$$

The formula (7) on both sides divided by the nominal area A, and then cleaning it can get

$$
\sigma^{0}=\frac{A^{\mathrm{f}}}{A} \sigma^{f}+\frac{A^{\mathrm{s}}}{A} \sigma^{s}
$$

Here $\sigma^{0}, \sigma^{f}$,and $\sigma^{s}$ respectively for the apparent stress, flow stress and solid contact stress (as shown in figure 2 (c)), and $\mathrm{D}$ means disturbance,

$$
D=\frac{A^{S}}{A}
$$

In the initial pressure, because of the contact area is too small to think about it, so $D \approx 0$.deformation, the contact between the particles tends to increase $D \approx 1$. Thus when the $\mathrm{D}=1, \sigma^{0}=\sigma^{s}$. when the $0<\mathrm{D}<1$, The full stress $\sigma^{0}$ shows the change that the sum of contact stress $D \sigma^{s}$ and the fluid stress $(1-D) \sigma^{f}$.In the process of deformation and the water out of porous material, the contact area $A^{s}$ increases, the liquid area $A^{f}$ is reduced. Thus , the stress $D \sigma^{s}$ that contact particles bear is increased , and the stress $(1-D) \sigma^{f}$ that liquid bear is reduced. ${ }^{[6-11]}$

\section{Conclusion}

It's the above derivation process simply introduce the perturbation equation in the dissimilar materials units, which is in the analysis of duality disturbance, and simply introduces the duality disturbance of saturated porous medium. In conclusion,in the theory of duality disturbance concept of varieties of material combination, It is possible that material is given different characteristics to simulate the behavior of the material unit (such as elastic, elastic-plastic, viscoelastic and viscoplastic).

\section{Acknowledgements}

This work was financially supported by the Jiangxi Science Foundation (20132BAB216007), scientific research fund of Jiangxi provincial education department (GJJ13440) and scientific research fund of East China Institute of Technology (DHBK201109).

\section{References}

[1] Jinhe Gao, Shoulong Zhang , Yanbin Huang, A Measurement Method of Tensile Strength for Irregular Small Stone, ICCET 2013, pp281-285, Kunming, 2013.12.14-15.

[2] Jinhe Gao, Yanbin Huang, Shoulong Zhang, Mechanic Model of Push-Out Test of Over-Thick CFST, 2014 International Conference on Advances in Materials Science and Information Technologies in Industry ( AMSITI 2014), pp314-318, Xian, 2014.1.11-12.

[3] Li Y Y, Cui J Z. The multi-scale computational method for the mechanics parameters of the materials with random distribution of multi-scale grains $[\mathrm{J}]$. Composites Science and Technology, 2005, 65 ( 9):1447-1458.

[4] Liu Jiemin, SUN Yazhen. Prediction of Mechanical Behavior of Equivalent Adhesive- Layer Using Theory of Duality of Damage [C]. New York: ASME, 2006.

[5] Sun Yazhen, Liu Jiemin. Coupling Analysis of Fracture Mechanics and Damage Mechanics for Fiber-Reinforced Asphalt Concrete Pavements [C]. New York:ASME. 2006.

[6][1]Desai C.S. Mechanics of Materials and Interfaces. CRC Press LLC, 2001

[7]Ferdinand P.B. Johnston Jr. Mechanics of Materials, New York:McGraw-Hill Inc.,1992

[8]A.A.Becker.Finite Element Analysis. New York:ASME,2004

[9]Terzaghi K., Peck R.B., Mesri G.Soil Mechanics in Enginerring Practice, A Wiley-Interscience

Publication(Third Edition),John Wiley\&Sons,1996. 
[10] Kang-Ning Li, "Analysis Model for 3D RC Columns Coupled with Shear Nonlinearity," Proceedings of the 9th Japan Earthquake Engineering Symposium, Vol.3, December 1994, pp. E-127-E-132.

[11] Kang-Ning Li and Tetsuo Kubo, "Reviewing the Multi-Spring Model and Fiber Model," The 10th Japan Earthquake Engineering Symposium, 1998, Volume 2, pp.2369-74.

[12] K. Li and X. Ye, "Equilibration of Element with Multi-Deformation Components for Nonlinear Structural Analysis", the 4th International Conference on Urban Earthquake Engineering, Tokyo Institute of Technology, Japan, March 2007. 